\title{
Habiter et types d'habitat à Alger
}

\author{
Nora Semmoud \\ Professeure Equipe Monde Arabe et Méditerranée \\ UMR 7324 CITERES/CNRS/Université François Rabelais Tours
}

\section{Résumé}

Le présent travail rend compte des stratégies d'appropriation de l'habitat par la population algéroise et les types qu'elles configurent. La typologie ainsi produite établit la structure de correspondance entre les dispositions spatiales d'un édifice et les conditions d'appropriation de ses occupants. L'appropriation de l'habitat est envisagée comme un processus de mise en conformité des lieux avec l'habitus des individus. Un processus où ils sont contraints de composer avec le contexte spécifique dans lequel ils le font, notamment avec les conditions de production de l'habitat. Les adaptations et les rectifications opérées font apparaître précisément l'ampleur des décalages entre propriétés spatiales de leur demeure et leurs modèles socioculturels. De cette façon, les pratiques d'appropriation restituent, d'une part, des types d'habitat disqualifiés par les individus et considérés comme transitoires et d'autre part, ceux qui émergent comme leur préférence.

\section{Mots clés}

Appropriation, usage, production de l'espace, habitat, types d'habitat, recomposition de l'espace.

Le présent travail rend compte d'une recherche ${ }^{1}$ sur les stratégies d'appropriation de l'habitat et de la ville par la population algéroise et les types ${ }^{2}$ qu'elles configurent. La typologie $^{3}$ que nous cherchons à construire est celle qui établit la structure de correspondance entre les dispositions spatiales d'un édifice et les conditions d'appropriation de ses occupants. Cependant aujourd'hui, les cartes apparaissent brouillées sous l'effet conjugué de deux facteurs. Le premier est que la population algéroise s'est appropriée à l'indépendance un parc immobilier qui ne lui était pas destiné. Le deuxième est que le produit logement du mouvement moderne et ses récents avatars ne restituent plus les correspondances qui intéressent notre problématique. Dans cette optique, des populations différentes, habitant un même type de bâti, se distinguent par leurs modalités d'appropriation de l'espace. Inversement

\footnotetext{
${ }^{1}$ La présente contribution actualise et complète en partie une recherche effectuée en 1993 et publié sous forme d'ouvrage chez l'Harmattan en 2001, Les Stratégies d'appropriation de l'espace à Alger.

2 Le type ici est une abstraction, il est entendu comme structure de correspondance entre les dispositions spatiales d'un édifice et les valeurs différentielles que lui attribue le groupe social auquel il est destiné (C. DEVILLERS, 1974).

3 Il ne s'agit pas de la typologie architecturale proprement dite, mais d'une abstraction des reconfigurations induites par les pratiques d'appropriation des habitants. Ainsi, ce n'est pas l'habitat illicite ou celui de la Casbah qui sont disqualifiés aux yeux des occupants, mais plutôt le mode d'appropriation auquel ils contraignent de nombreux ménages (maison commune ou familiale).
} 
des conditions d'appropriation similaires peuvent prendre effet dans des types de bâtis différents. Par conséquent, afin d'identifier les types d'habitat, entendus comme structure de correspondance entre spécificités des habitants et organisation architecturée des lieux, nous avons déplacé nos investigations sur le terrain passionnant des phénomènes d'appropriation entendus comme :

«[...] l'ensemble des actions des hommes dans l'espace, consistant simultanément à lui donner des configurations spatiales matérielles et des significations. » (H. Lefebvre, 1970, p. 203)

L'appropriation de l'espace par les individus permet de saisir la confrontation dans l'espace entre les pratiques de l'urbanistique et celles de la population et ainsi de mettre en évidence la dialectique qui existe entre les conditions de production et d'usages de l'espace. Elle autorise en outre l'accès aux formes de recomposition sociale de l'espace, tant dans la construction sociale des quartiers que dans les rectifications opérées sur les dispositions spatiales de l'habitat. Ces processus d'adaptation et de rectification font apparaître différents types d'habitat, certains sont rejetés par leurs occupants et considérés comme transitoires tandis que d'autres deviennent des modèles dominants.

Le terrain passionnant des phénomènes d'appropriation fait écho à celui des processus d'actualisation de l'habitus décrit par Pierre Bourdieu (1972), des processus qui transforment, adaptent, retournent ou annulent un espace. En fait, les habitants n'actualisent leur habitus qu'en composant avec un cadre physique particulier. Etant entendu que les schèmes pratiques à la base de cette actualisation, quelle que soit leur inertie, subissent des mutations qui apparaissent à l'occasion du décodage des significations des modalités d'usage. Le processus de réappropriation ou de mise en conformité des lieux avec l'habitus représente également des stratégies pour dépasser les obstacles dressés par la violence symbolique ${ }^{4}$. Ainsi, chez Pierre Bourdieu, le concept d'actualisation des habitus désigne cette confrontation entre habitus et violence symbolique. Dans cette optique, il faut souligner l'apport considérable d'une école marxisante représentée par Henri Lefebvre qui, à notre sens, a rendu compte de la complexité $\mathrm{du}$ concept d'espace entendu dans sa dimension pratico-symbolique. L'auteur introduit en particulier une distinction importante entre ce qui se passe dans l'espace, qui relève du vécu et du perçu par les habitants, et ce qui est fait de l'espace par les professionnels de l'aménagement (H. Lefebvre, 1970, p. 46). Ensuite, au sein des rapports que les divers groupes sociaux 5 entretiennent avec l'espace physique, il y a l'espace des représentations mentales qui véhicule une série de symboles et d'images. L'appropriation de l'espace se fait alors au travers de l'imagination et de l'utilisation symbolique des objets de l'espace physique. Ces représentations de l'espace et les pratiques qu'elles supposent créent un système de signes et de symboles, auquel il faut nécessairement accéder pour saisir le sens des recompositions sociales opérées par les actions quotidiennes des groupes sociaux. Ces images et ces symboles constituent vraisemblablement les représentations les plus riches que peut avoir une société de son espace.

Aborder les phénomènes d'appropriation de l'habitat à Alger suppose inévitablement de tenir compte des difficultés de nombreux citadins à se loger convenablement et de leurs incidences

\footnotetext{
4 «[...] l'espace est un lieu où le pouvoir s'affirme et s'exerce, et sans doute sous la forme la plus subtile, celle de la violence symbolique comme violence inaperçue: les espaces architecturaux, dont les injonctions muettes s'adressent directement au corps, obtenant de lui, tout aussi sûrement que l'étiquette des sociétés de cour, la révérence, le respect qui naît de l'éloignement ou, mieux, de l'être-loin, à distance respectueuse, sont sans doute les composantes les plus importantes, en raison même de leur invisibilité [...], de la symbolique du pouvoir et des effets tout à fait réels du pouvoir symbolique. " (P. BOURDIEU, 1993).

5 Le groupe social est entendu ici comme un ensemble de personnes ayant des caractères, des relations et des sentiments communs. COUET (Jean-François) et DAVIE (Anne), 2002, (3 $3^{\text {ème }}$ éd.), Dictionnaire de l'essentiel en sociologie, Paris, Editions Liris.
} 
sur les pratiques individuelles et collectives. Dans ce cas, les significations des phénomènes d'appropriation traduisent autant les modèles culturels de l'habitant que les stratégies mises en œuvre pour gérer la pénurie d'espace. A propos de la question du logement, nous renvoyons aux réflexions de Sid Boubekeur (1986), parce qu'elles établissent une rétrospective critique des différentes thèses sur le sujet. Selon l'auteur (1986, p. 11), la crise du logement renvoie à la fois à une crise de développement, ainsi qu'en témoigne le processus rapide d'urbanisation et à une crise de société car les bouleversements des modes de vie des habitants et de leurs représentations sociales ont défini des besoins nouveaux, notamment la préférence pour les logements modernes et les matériaux lourds comme le béton et la brique. Le ministère estime le déficit à 1,127 millions logements 6 , sans compter les besoins liés à l'accroissement démographique considéré parmi les plus élevés du monde. Il n'en demeure pas moins que cette situation doit être nuancée car comme le souligne à juste titre André Prenant (2002) parler d'une «crise du logement » à Alger relèverait d'un «consensus » général qui masque la réalité. Cet auteur s'appuie sur le dernier recensement ${ }^{7}$ pour montrer que la distorsion entre le nombre des ménages et les locaux d'habitation a considérablement diminué, que le TOP 8 a baissé et surtout qu'il existe aujourd'hui un important parc de logement inoccupés ${ }^{9}$. Par conséquent, la question du logement relève moins d'un décalage entre l'offre et la demande que des difficultés rencontrées par les groupes sociaux défavorisées à faire valoir leur droit au logement. La mise en œuvre de programmes de logements sociaux 10 (locatifs ou d'accession sociale ${ }^{11}$ ) contribue sans doute à baisser la tension, mais il semble que des pratiques de détournement continuent à peser dans l'exclusion des ménages les plus modestes. Il est vrai que nos investigations portent uniquement sur Alger, cependant, sans généraliser à tout prix, la capitale, en tant que centre diffuseur de modèles, peut être, dans une certaine mesure, révélatrice des transformations socioculturelles qui touchent, à plus ou moins brève échéance, le pays dans son ensemble.

\section{Logiques d'appropriation et types d'habitat.}

L'objectif de ce travail est de mettre en évidence les types 12 d'habitat que les individus dessinent par leurs appropriations du logement produit. Ainsi, la seule façon d'identifier les

\footnotetext{
${ }^{6}$ Chiffres publiés par le ministère de l'urbanisme et de la construction et de l'habitat au sein de plusieurs quotidiens au mois de février 1994.

7 RGPH 1998.

8 Taux d'occupation par pièce est passé de 2,43 en 1966 à 2,22 en 1998.

9 Plus de 49200 logements vacants (logements inoccupés selon l’ONS), soit 11,7\% du parc total recensé en 1998. Entre 1987 et 1998, l'accroissement des logements vacants s'est particulièrement manifesté dans la périphérie algéroise, là où se situe l'essentiel des réalisations en matière de logements. Ainsi, par exemple le nombre de logements vacants s'est accru de $73 \%$ à Bir Mourad Raïs (Sud-Ouest).

10 Selon le ministre de l'habitat, Mohamed Nadir Hamimid, 248107 logements sociaux locatifs et 107217 logements sociaux participatifs ont été livrés en 2004. Numéro spécial du quotidien El Ahrar du $1^{\text {er }}$ novembre 2004.

11 Il s'agit des opérations LSP (logement social participatif).

12 Le type devient représentatif d'une classe d'édifices ayant en commun les propriétés formelles les plus significatives des pratiques spatiales du groupe social destinataire. Dans cette optique, Jean-Pierre Frey (1991, p. 52) place le type dans la même perspective de conceptualisation qu'Henri Raymond et l'envisage dans le cadre d'une théorie qui rend compte des conditions sociales d'existence. Autrement dit, le type est contenu dans la relation entre le mode de conception et de production d'un édifice et les caractéristiques sociales, culturelles et économiques de son usage par l'habitant. Cependant, cette relation n'est effective qu'à certaines périodes historiques, celles pour lesquelles le programme architectural correspond explicitement aux spécificités des groupes sociaux visés. On identifie ainsi, au cours de l'histoire, une succession d'édifices typiques d'un groupe social, tels que la villa bourgeoise, l'hôtel particulier du XVIII, l'immeuble haussmannien... La conception du mouvement moderne de l'usager en tant qu'être de "besoins" plutôt que de "pratiques" a gommé les différences sociales et culturelles perceptibles au niveau de la forme en uniformisant et standardisant le produit architectural. Depuis, la réflexion autour du type architectural s'est imposée comme la seule voie pour lever les obstacles à la compréhension et à la prise en compte des pratiques d'appropriation. En effet, à travers le travail sur le type, remarque Pierre Pinon (1991, p. 57),
} 
types d'habitat, en tant que structure de correspondance entre spécificités des habitants et organisation architecturée des lieux, est de déplacer les investigations sur le terrain des phénomènes d'appropriation. Le décodage des significations (sociales, imaginaires, symboliques, etc.) contenues dans le discours des habitants et dans le marquage de l'espace induit par leurs modalités d'appropriation (investissement, ameublement, décor, affectation des pièces, spécialisation, fréquentation, système d'opposition 13 , etc.) permet en définitive d'identifier les modes 14 d'habiter.

"L'habitat [ou l'espace en général] n'est pas un objet inerte, composé d'éléments techniques et doté de formes, mais un "objet actant", mis en mouvement, malgré son apparence statique, par les personnes qui l'utilisent. Il n'existe qu'à travers les interactions qui se développent entre ses éléments fonctionnels et ses occupants, qui lui confèrent tout son sens. Inversement, bien que chacun transporte d'un lieu à l'autre ses façons d'agir, ses valeurs, ses sentiments et ses pensées profondes, ses conduites sont influencées par l'espace dans lequel elles se déploient, elles s'actualisent de manière spécifique selon les lieux qui leur servent de cadre »(M. Bonetti, 1994, p.16)

L'analyse se structure selon deux volets : le premier porte sur la relation entre le mode de conception/production du logement et les caractéristiques économiques et sociales de l'habitant - maître d'œuvre. Le second volet traite des formes d'appropriation de logements non produits par leurs habitants. En conséquence de quoi la correspondance entre les phénomènes d'appropriation et les caractéristiques spatiales met au contraire en évidence les décalages entre conception et usage, quand ce dernier arrive à s'exprimer. Dans ce cas, les groupes sociaux ont une identité contrariée par les difficultés à signifier leur spécificité à travers des types d'édifices et leur contexte urbain. Les pratiques d'appropriation rendent compte des stratégies de la population et des activités multiples qu'elle initie, afin d'adapter le logement à ses spécificités et de compléter le produit logement pour en faire son habitat, de rétablir les articulations entre l'espace domestique et l'espace urbain et enfin de prétendre à une intégration à la ville. C'est au cours de ces processus de production ou d'adaptation de l'habitat qu'apparaissent les spécificités des groupes sociaux, leurs niveaux différentiels d'expression, ainsi que leur degré d'intégration à l'urbain (J.P. Frey, 1986).

$\mathrm{Au}$ cours des processus d'actualisation de l'habitus, le génie et les compétences 15 des habitants sont fonction précisément de l'ampleur du décalage entre les propriétés spatiales de leur logement et leurs modèles socioculturels. Force est de constater que le trajet de la mise en conformité de l'espace physique avec l'habitus est d'autant plus long et semé d'obstacles qu'il concerne des groupes sociaux modestes. C'est d'ailleurs ce milieu social qui développe les modalités d'appropriation les plus laborieuses et les plus remarquables, notamment celles qui consistent à gérer la pénurie d'espace et à protéger les intimités multiples au sein de l'espace domestique. En revanche, les groupes sociaux aisés qui ne sont pas a priori obligés de composer avec un espace qui leur est imposé, dépassent les contraintes en faisant jouer leurs

c'est la pratique des espaces qui est respectée et cette optique de la recherche sur le type ouvre un horizon nouveau à la conception architecturale.

13 Le système d'opposition, emprunté à Nicole HAUMONT (2001), renvoie à une organisation de l'espace domestique selon les oppositions : public/privé, derrière/devant, caché/montré, dehors/dedans, etc.

14 La recherche opère une translation à partir d'une typologie de onze modèles architecturaux algérois définis par le sens commun (l'habitat de la Casbah, des cités de recasement, des lotissements illicites denses, des lotissements illicites de standing moyen, des HLM du Plan de Constantine, des HLM de la période post-indépendante, des lotissements de l'époque coloniale, des nouveaux lotissements, Immeubles haussmanniens, des résidences du mouvement moderne, des ensembles récents de standing), vers une taxinomie de huit modes d'habiter qui finalement configurent 4 types d'habitat: la maison commune, la maison familiale, l'immeuble familiale modeste et l'immeuble familiale riche.

15 Ce concept est largement développé dans l'ouvrage dirigé par Isabelle Berry-Chikhaoui et Agnès Deboulet, paru en 2000. 
moyens. Lorsque la maison ne convient pas, on engage des transformations coûteuses ou on en construit une autre plus grande ou plus adaptée. Si les pratiques d'appropriation reflètent les spécificités des groupes sociaux, elles permettent aussi de situer où ils en sont dans leur mobilité sociale.

En définitive, les pratiques d'appropriation formalisent de nouvelles dispositions d'ensemble qui restituent autant les éléments spatiaux qui se prêtent le mieux aux spécificités des groupes sociaux que ceux qui les contrarient. Dans cette dynamique apparaissent alors les types d'habitat qui émergent en tant que préférence, par l'image positive qu'ils reflètent et ceux qui sont, au contraire, disqualifiés par les habitants. Les premiers qui correspondent à l'immeuble familial, modeste ou huppé, se prêtent le mieux au désir d'habiter de la population et deviennent l'objectif à atteindre pour de nombreux ménages. En revanche, il peut arriver que le logement de départ limite les améliorations, quels que puissent être les efforts consentis par les habitants. Ces types de logement qui correspondent à une appropriation selon la maison commune ou à la maison familiale censurent les habitus et contrarient les pratiques d'appropriation à tel point qu'ils ont toujours un caractère transitoire aux yeux de leurs occupants.

\subsection{Les types d'habitats disqualifiés}

En même temps que des types d'habitat émergent et deviennent des modèles dominants, d'autres, malgré toute la compétence des habitants à les transformer, restent loin de correspondre aux spécificités des groupes sociaux. Les modalités d'appropriation y sont particulièrement contrariées, l'espace domestique se prête mal aux transformations souhaitées pour acheminer l'habitat vers une organisation et une image satisfaisante et les familles n'envisagent son occupation que de façon transitoire. A ce titre, la maison commune est souvent l'origine du processus d'urbanisation. Dans la maison commune où chaque pièce est occupée par une famille différente, sans lien de parenté avec ses voisines, l'appropriation consistera à lever les contraintes liées à la cohabitation forcée avec des étrangers, à gérer la pénurie d'espace et à protéger les intimités multiples au sein d'une même famille, en procédant à un compartimentage des pièces voire de l'unique pièce/logement. Ce type d'habitat disqualifiant les occupants et disqualifié par ces derniers aura en permanence un caractère provisoire aux yeux des ménages qui mobiliseront toutes leurs stratégies pour quitter les lieux. Ce type apparaît à travers l'appropriation des maisons de la Casbah ${ }^{16}$, des cités de

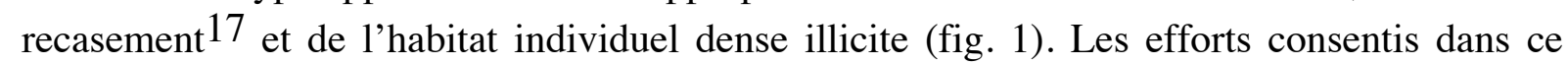
type d'habitat par les ménages reflètent une population modeste constituée généralement par des manœuvres ${ }^{18}$, des ouvriers, des employés et de nombreux chômeurs.

\footnotetext{
16 La Casbah (médina d'Alger) est depuis longtemps le lieu d'accueil et de transit des populations de l'intérieur du pays.

17 L'organisation spatiale des cités de recasement, conçues dans le cadre du Plan de Constantine (1958/1962), s'inspirait de celle des bidonvilles et se caractérisait généralement par 2 petites pièces donnant sur une cour (J. De MAISONSEUL, 1980).

18 Ce sont généralement les catégories socioprofessionnelles qui apparaissent à l'occasion de notre enquête.
} 


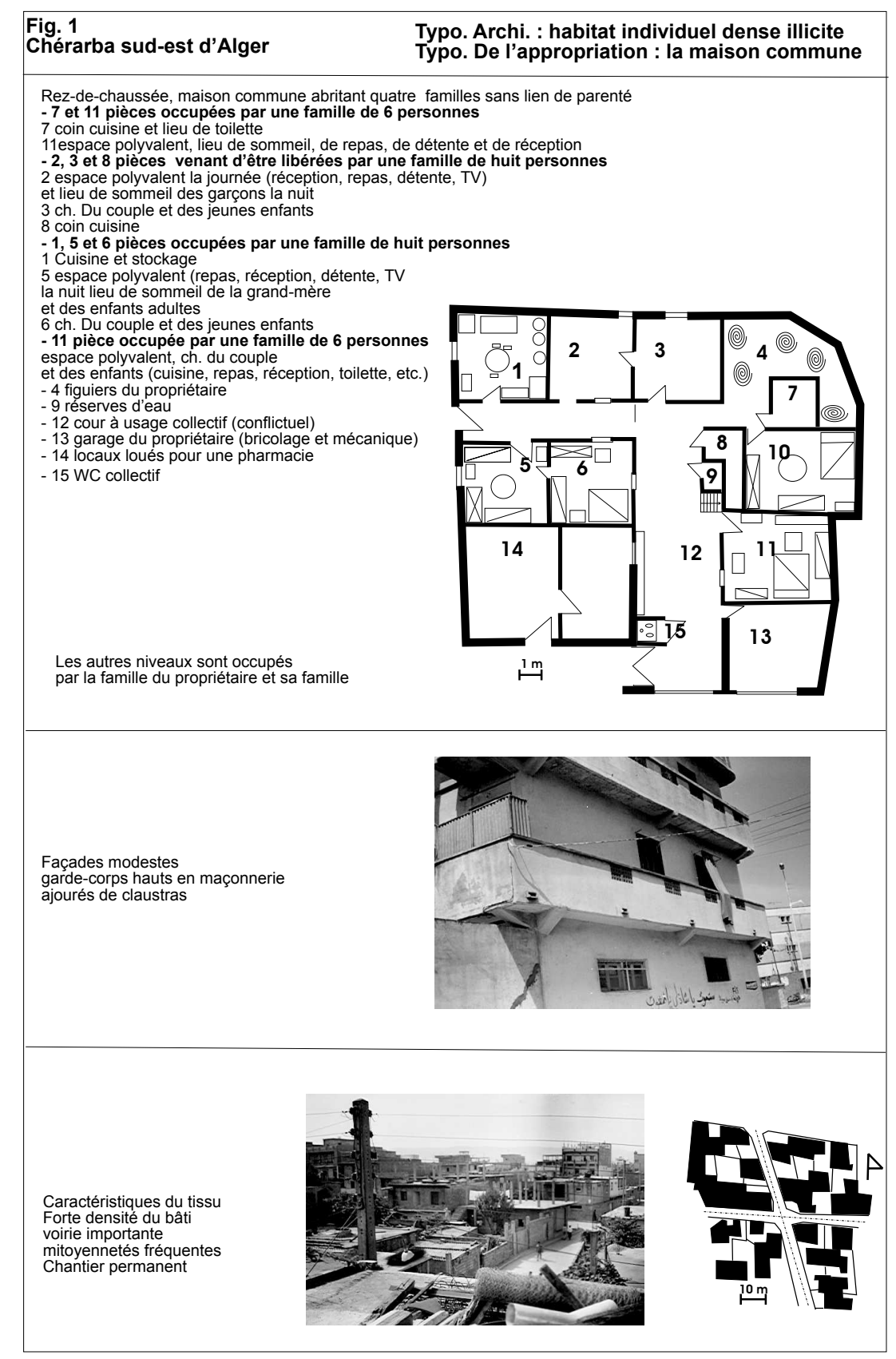

La maison commune devient maison familiale lorsque les pièces de cet habitat, organisé généralement autour d'une cour, sont occupées chacune par des ménages de la même famille. Si la maison familiale contrairement à la maison commune permet la protection des intimités multiples au sein de la famille, elle est toutefois loin de correspondre à l'idéal d'indépendance des couples. Il faut relever le statut de compromis transitionnel conféré à la maison de 
cohabitation intergénérationnelle (maison familiale), où l'espace du couple est tout de même individualisé. Les solidarités qu'implique cette forme d'habitat permettent soit au couple de thésauriser pour acquérir son propre logement, soit à la famille de se constituer les moyens pour édifier l'immeuble familial. La maison familiale prend effet tant dans l'habitat individuel, illicite ou celui des cités de recasement, que dans l'habitat collectif comme les HLM. La population reste généralement modeste mais se différencie de la première par l'apparition de nouvelles catégories socioprofessionnelles comme les techniciens, les cadres moyens et les commerçants. Bien que l'idéal-type des habitants des HLM soit l'immeuble familial, leurs espérances se limitent à obtenir un appartement plus grand dans des ensembles collectifs à image valorisante. Ils intègrent de facto que leur condition acquise ou ambitionnée ne leur permettrait pas de construire leur propre habitat et encore moins l'immeuble familial.

CORRESPONDANCES : TYPE D'HABITAT, APPROPRIATIONS, ARCHITECTURES

\begin{tabular}{|l|l|l|}
\hline $\begin{array}{l}\text { Types } \\
\text { disqualifiés et } \\
\text { transitoires }\end{array}$ & La maison commune & La maison familiale \\
\hline $\begin{array}{l}\text { Catégories } \\
\text { socio } \\
\text { professionnelle } \\
\mathbf{s}^{19}\end{array}$ & $\begin{array}{l}\text { Chômeurs, manœuvres, } \\
\text { ouvriers et employés }\end{array}$ & $\begin{array}{l}\text { Chômeurs, ouvriers qualifiés, } \\
\text { employés, techniciens, cadres moyens } \\
\text { et commerçants }\end{array}$ \\
\hline $\begin{array}{l}\text { Logiques } \\
\text { d'appropriatio } \\
\text { n }\end{array}$ & $\begin{array}{l}\text { Gestion de la cohabitation } \\
\text { avec des étrangers et } \\
\text { protection des intimités }\end{array}$ & $\begin{array}{l}\text { 1) Densification et dégradation } \\
\text { 2) Gestion de la pénurie d'espace }\end{array}$ \\
\hline $\begin{array}{l}\text { Typo. } \\
\text { architecturale }\end{array}$ & $\begin{array}{l}\text { 1) L'habitat de la Casbah } \\
\text { 2) Les cités de recasement } \\
\text { 3) L'habitat individuel dense } \\
\text { (illicite) }\end{array}$ & $\begin{array}{l}\text { 1) L'habitat individuel dense (illicite) } \\
\text { 2) Les cités de recasement } \\
\text { 3) Les HLM du Plan de Constantine } \\
\text { 4) Les logements sociaux de la période } \\
\text { post-indépendante20 }\end{array}$ \\
\hline $\begin{array}{l}\text { Typo. du sens } \\
\text { commun } 21\end{array}$ & $\begin{array}{l}\text { 1) Diar ara'b } 22 \\
\text { 2) Bénian faùdawi } 23\end{array}$ & $\begin{array}{l}\text { 1) Bénian faùdawi } \\
\text { 2) HLM }\end{array}$ \\
\hline
\end{tabular}

\subsection{Les types d'habitats valorisants}

Quelque que soit le groupe social concerné, une préférence très nette apparaît pour ce que nous appelons l'immeuble familial 24 (fig. 2). Son organisation spatiale se caractérise par la

\footnotetext{
19 Il s'agit des catégories socioprofessionnelles repérées à l'occasion des enquêtes que nous avions effectuées dans ces quartiers.

20 Il est vrai que les jeunes couples aspirent au logement social pour sortir de la cohabitation familiale et de nombreux ménages ont pu «se desserrés » grâce à la réalisation récente de nombreux logements sociaux, cependant les conditions d'accès restent encore ségrégatives et excluent les ménages les plus démunis qui sont contraints à utiliser l'appartement comme une maison familiale.

21 Il s'agit d'une typologie que Jean-Pierre FREY (1991, p.52) qualifie de spontanée, véhiculée par le langage et la culture portés par les habitants. Enquête menée par les étudiants de l'EPAU en 1990 auprès de 127 ménages algérois dans différents quartiers, autour de la typologie de l'habitat.

22 Maisons arabes.

23 Constructions anarchiques.
} 
superposition de plusieurs logements, plus ou moins indépendants dans un immeuble implanté sur un même lot où cohabitent dans des appartements séparés, les parents et les jeunes ménages des fils. Généralement la cage d'escalier et les espaces extérieurs, tels que la cour, le jardin et la terrasse sont utilisés en commun, particulièrement au moment des fêtes familiales. Selon la richesse de la famille, d'autres espaces communs apparaissent, comme le sous-sol, la cave et le hammam. Les résidents de cet d'habitat l'évoquent avec fierté comme l'accomplissement de leur réussite sociale, tandis que les groupes sociaux qui n'y ont pas accès, en parlent comme l'idéal-type à atteindre. Cette position privilégiée de l'immeuble familial dans les représentations des groupes sociaux est due à trois conditions très recherchées, le statut de propriétaire, le caractère individuel de l'habitat et la latitude qu'il offre aux usagers, notamment d'en faire une source de revenu 25 et enfin, la possibilité de léguer un patrimoine aux enfants, surtout aux fils.

Le statut de propriétaire octroie de facto à son titulaire une place parmi les possédants et, par conséquent, la possibilité de léguer un héritage. Cette place dans l'échelle sociale est perçue par l'ensemble du corps social comme étant stable et sécurisante. Cependant, de nombreux ménages, qui ont opté pour l'immeuble familial avouent qu'ils auraient préféré offrir à leurs héritiers des logements totalement indépendants qui auraient permis aux couples de vivre de façon autonome. Toutefois les coûts prohibitifs de l'immobilier réservent ces possibilités uniquement aux groupes sociaux les plus aisées. Ainsi de ménages ont produit ce type d'habitat par leurs tentatives de lever les obstacles auxquelles les exposent les caractéristiques du marché immobilier actuel.

\footnotetext{
24 Il est vrai que l'immeuble familial existe depuis longtemps, mais ce travail cherche à montrer son émergence en tant que modèle dominant chez les ménages.

25 Les sources de revenus que permet ce type d'habitat peuvent être la location de logements et de garages ou l'installation par la famille de sa propre activité économique.
} 


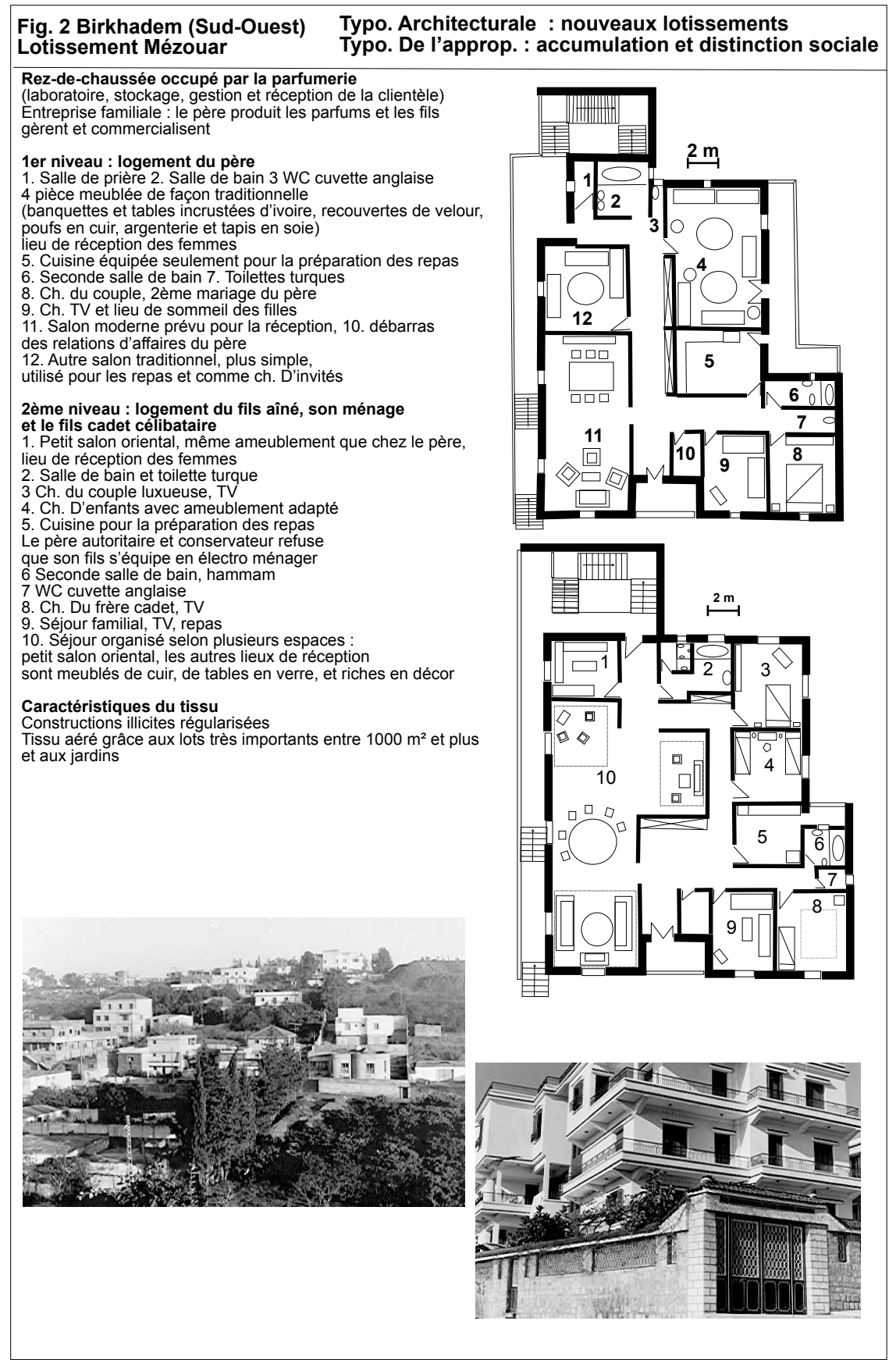

La volonté quasi générale de protéger l'indépendance des couples se manifeste dans les formes d'organisation spatiale de ces immeubles familiaux, notamment par l'emplacement des espaces de distribution commun à l'extérieur des appartements privés. Ces espaces de 
distribution, qui englobent accès, cage d'escalier, hall et paliers, prennent à ce titre le statut de lieux typiques partiels. Lorsque ces derniers se trouvent à l'intérieur des appartements et, de ce fait, ne permettent pas une vie indépendante des différentes entités familiales, l'un des soucis majeurs partagé par tous est de les déplacer à l'extérieur de l'espace habitable. Les femmes, particulièrement sensibles aux possibilités d'indépendance ainsi offertes, trouvent dans ce type d'habitat les avantages d'un voisinage familial, mais pas les inconvénients de la maison familiale où la proximité avec les autres ménages est contraignante pour le couple. Les bellesfilles, par exemple, soulignent le sentiment de sécurité que procure l'immeuble familial, car il suppose solidarité et entraide familiales mais apprécient la distance maintenue avec les beauxparents, en particulier les belles-mères, dont le poids contrarie souvent l'épanouissement du couple. D'un autre coté, les parents apprécient d'avoir leurs enfants et petits-enfants à proximité, sans avoir à supporter dans une proximité plus grande le mode de vie des jeunes avec lequel ils sont plutôt en désaccord. A Birkhadem, cette logique familiale a donné lieu à un lotissement familial : un ensemble de villas organisé autour d'une impasse privée (fermée par un portail) et occupé exclusivement par les membres d'une même famille (frères, sœurs, cousins, parents, oncles, etc.).

La question de savoir si ce type d'habitat, actuellement dominant dans les choix résidentiels des ménages, est transitoire ou non reste posée. Il est vrai que l'amplification de la mobilité résidentielle due aux itinéraires professionnels aléatoires et la diminution de la tension qui caractérise le marché immobilier algérien peuvent remettre en cause les fondements de ce type d'habitat. Mais l'inertie des modèles d'habiter fera de l'immeuble familial un type durable, même s'il perd son caractère dominant. Dans tous les cas, il traduit une solidarité familiale pour loger décemment les différents ménages et apparaît comme un compromis face aux aléas du marché immobilier.

A notre sens, la mobilité professionnelle des héritiers et la libéralisation du marché du logement feront disparaître progressivement l'immeuble familial chez les groupes sociaux les plus nantis. Toutefois, le modèle de l'immeuble familial sera repris par des milieux plus larges qui le considèrent aujourd'hui comme l'idéal-type à atteindre. Pour ces groupes sociaux au revenu modeste, l'immeuble familial correspond à une volonté d'échapper à une situation sociale vulnérable.

Dans notre analyse, l'immeuble familial a évidemment des formes différentes selon les groupes sociaux. Les couches moyennes supérieures, comme les cadres, les hauts fonctionnaires et les professions libérales y signifient, notamment par les pratiques du «grand», de l'accumulation et de l'ostentation, leur richesse, tandis que l'architecture de l'immeuble familial des groupes sociaux aux revenus moins élevés présente moins de prétentions. L'immeuble familial modeste, en revanche, évolue et s'adapte aux besoins de la famille en même temps qu'il permet de gérer sa mobilité sociale par le truchement d'une activité commerciale ou de service. Son caractère évolutif 26 confère au quartier l'aspect d'un chantier permanent. Les catégories socioprofessionnelles dominantes dans ce type d'habitat sont les ouvriers qualifiés, les cadres moyens, les commerçants et les artisans.

\section{CORRESPONDANCES TYPE D'HABITAT, APPROPRIATION, ARCHITECTURE.}

\footnotetext{
26 Il y a une sorte de synchronie entre la montée en puissance de l'activité économique, les transformations de l'habitat et la mobilité sociale du ménage : dans un $1^{\text {er }}$ temps, c'est souvent la mise en route de l'activité économique, dés le début de la construction, qui permet de la financer. Dans un $2^{\text {ème }}$ temps, lorsque cette activité atteint son rythme de croisière, elle permet la réalisation d'un autre niveau. Dans un $3^{\text {ème }}$ temps, l'accumulation générée va permettre un mariage et ainsi la réalisation d'un autre appartement, etc.
} 


\begin{tabular}{|c|c|c|}
\hline $\begin{array}{l}\text { Types } \\
\text { d'habitat, } \\
\text { modèles } \\
\text { dominants }\end{array}$ & $\begin{array}{l}\text { Immeuble familial modeste, } \\
\text { articulation habitat / activité }\end{array}$ & $\begin{array}{l}\text { Immeuble familial riche, } \\
\text { articulation habitat /activité }\end{array}$ \\
\hline \begin{tabular}{|l|} 
catégories \\
socio \\
professionnelle \\
S
\end{tabular} & $\begin{array}{l}\text { Ouvriers qualifiés, cadres moyens, } \\
\text { commerçants, artisans. }\end{array}$ & $\begin{array}{l}\text { Cadres, fonctionnaires, professions } \\
\text { libérales, commerçants et chefs } \\
\text { d'entreprise }\end{array}$ \\
\hline $\begin{array}{l}\text { Logiques } \\
\text { d'appropriatio } \\
\text { n }\end{array}$ & $\begin{array}{l}\text { 1. Mobilités sociales à partir de } \\
\text { l'habitat } \\
\text { 2. Processus d'adaptation aux } \\
\text { besoins }\end{array}$ & $\begin{array}{l}\text { 1. Accumulation et distinction } \\
\text { sociale } \\
\text { 2. Investissements familiaux } \\
\text { dépassant les capacités du } \\
\text { logement } 27\end{array}$ \\
\hline $\begin{array}{l}\text { Typologie } \\
\text { architecturale }\end{array}$ & $\begin{array}{l}\text { 1. Habitat individuel dense } \\
\text { 2. Lotissements modestes de } \\
\text { l'époque coloniale }\end{array}$ & $\begin{array}{l}\text { 1. Nouveaux lotissements } \\
\text { 2. Habitat individuel à } \\
\text { standing moyen } 28 \\
\text { 3. Immeubles haussmanniens } \\
\text { 4. Résidences du mouvement } \\
\text { moderne } \\
\text { 5. Ensembles récents de } \\
\text { standing }\end{array}$ \\
\hline $\begin{array}{l}\text { Typo. du sens } \\
\text { commun }\end{array}$ & $\begin{array}{l}\text { 1. Bénian faùdawi } \\
\text { 2. Diar wakt frança, diar el } \\
\text { colon }^{29}\end{array}$ & $\begin{array}{ll}\text { 1. } & \text { Villas } \\
\text { 2. } & \text { Batimat wakt frança } 30 \\
\text { 3. } & \text { Batimat djedad } 31\end{array}$ \\
\hline
\end{tabular}

\section{La recomposition sociale de l'urbain}

\subsection{Intégration d'une activité économique à l'habitat}

Les pratiques d'appropriation de l'habitat sont clairement destinées à affirmer une identité sociale acquise ou des prétentions. Elles apparaissent très diverses et la plus remarquable est celle qui consiste à organiser au sein de l'habitat individuel une activité à caractère lucratif dont les ressources permettent un appoint non négligeable dans les budgets familiaux ou offrent une opportunité pour lancer des activités pour les jeunes actifs sans emplois. L'intégration d'activités à caractère économique au sein de l'immeuble familial et plus généralement dans l'habitat individuel est également un fait spécifique remarquable. Il consiste à réserver un espace au sein de l'habitation (garage, sous-sol ou une partie du rez-dechaussée) pour organiser une activité à caractère lucratif, telle que commerce, artisanat, petites industries ou cabinet de profession libérale. L'habitat peut également être l'objet en partie d'un investissement locatif (dépôts divers, logements, bureaux, etc.). Cette aspiration quasi générale à articuler une activité à l'espace domestique, parce qu'elle est réalisable uniquement dans le cas de l'habitat individuel, milite largement en faveur de ce type d'habitat.

\footnotetext{
27 Ces ménages ont réaménagé plusieurs fois leur logement afin qu'il puisse recevoir l'ameublement, l'équipement et les éléments de décor, mais sa saturation engage les familles dans la construction.

28 Cet habitat correspond à l'urbanisation informelle de la périphérie algéroise des années 1970 et qui a été régularisé dans les années 1980. Il abrite des ménages aux revenus moins élevés que les bénéficiaires des nouveaux lotissements issus de programmes officiels des années 1980 destinés aux couches moyennes supérieures.

29 Les maisons du temps de la France, les maisons des colons.

30 Les bâtiments du temps de la France.

31 Les nouveaux bâtiments.
} 
L'importance de ce projet est soulignée par le fait que les constructeurs, au moment de choisir leur lot, écartent d'emblée ceux desservis exclusivement par une impasse. Celle-ci, en tant que desserte semi-privée est considérée comme un espace qui rebute les passants et par conséquent la clientèle potentielle. C'est un lieu peu avenant pour les étrangers au quartier qui se prête mal au stationnement de plusieurs véhicules et encore moins aux approvisionnements. C'est ainsi que l'impasse, traditionnellement privilégiée pour protéger l'habitat, ne sera plus une norme d'urbanisation des lotissements plus récents. Les habitants sont ainsi d'autant plus sensibles aux caractéristiques de la morphologie urbaine qu'ils accèdent à une maitrise plus grande de leur habitat et de leurs activités économiques dans l'espace urbain.

La différence de signification attribuée par les habitants à une activité économique dans le logement a constitué pour nous un indicateur précieux pour affiner notre analyse des groupes sociaux. Ainsi nous avons donc pu définir trois groupes sociaux qui se distinguent chacun par sa perception de cette pratique et le sens qu'il lui donne. Cette pratique est particulièrement visible dans l'habitat illicite et les lotissements communaux qui forment les quartiers populaires denses. Les habitants, dont le revenu familial reste insuffisant vu le nombre de personnes à charge, réservent systématiquement dans leur logement un espace qu'ils destinent à une activité commerciale et artisanale (mécanique, tôlerie, ferronnerie, etc.) ou encore immobilière. Pour les milieux populaires, l'activité doit permettre de sortir d'une situation de vulnérabilité sociale, notamment de parer au manque de travail et à une inflation galopante. Ce lieu d'activité est tellement important dans l'itinéraire social de la famille et ses projections sur l'avenir que les constructeurs entament d'abord la réalisation de cet espace avant l'achèvement de la maison. C'est souvent la mise en service rapide de l'activité et les revenus qu'elle procure qui permettent de poursuivre la construction. Ce phénomène s'est également généralisé dans les anciennes cités de recasement où les habitants, malgré les forts taux d'occupation par pièce, ont sacrifié un espace pour le destiner à un commerce ou une activité artisanale. Les familles qui n'ont pas pu concrétiser ce projet, notamment à cause de leur localisation dans une impasse, se distinguent des autres par leur précarité sociale. Ce sont les familles qui n'ont pas pu procéder, par exemple, à l'extension de leur habitat ou à l'achat d'une voiture. L'articulation d'une activité à l'habitat représente pour ce groupe social une condition indispensable, et souvent la seule, pour sortir d'une situation sociale précaire et prétendre à une ascension sociale.

L'insertion d'une activité au sein de l'habitat est une pratique que l'on retrouve également chez les groupes sociaux favorisés qui ont bénéficié des procédures de lotissement. Cependant, les ménages dans ce cas confèrent à cette pratique une signification différente de celle des milieux populaires. Dans ce cas de figure, l'espace réservé aux activités est plus important car il est conçu pour accueillir des entreprises industrielles (cosmétique, alimentaire, confection, etc.), une profession libérale ou un commerce de luxe. L'activité commerciale dans les nouveaux lotissements qui n'est pas limitée à l'alimentation générale, comme c'est souvent le cas dans les quartiers populaires, mais englobe également le prêt-à-porter, la pâtisserie et la bijouterie. Il s'agit en fin de compte de commerces destinés à la clientèle aisée du lotissement. Les catégories socioprofessionnelles qui ont bénéficié de ces nouveaux lotissements caractéristiques du paysage urbain actuel sont composées majoritairement de cadres, de fonctionnaires, de professions libérales, de chef d'entreprises et de commerçants. Correspondant à une volonté d'accumulation immédiate, ces activités échappent partiellement voire totalement au fisc et sont par ailleurs une source de nuisances pour le voisinage (bruits de machines, déchets industriels, etc.). Les conflits fréquents dans ces quartiers sont essentiellement liés à ces nuisances.

\subsection{L'extraversion de l'habitat}


Dans des quartiers où l'on tente d'échapper à la marginalisation urbaine et où l'on gère une insertion effective dans la société urbaine, les pratiques d'appropriation et les compétences des habitants pour échapper aux contraintes imposées et pour signifier leurs spécificités sociales font apparaître une tendance quasi générale à formaliser un type d'habitat caractérisé par une urbanité grandissante. Les types d'habitat qui émergent à travers les constructions récentes ainsi que les transformations qui touchent le parc ancien se caractérisent par une nette tendance à l'extraversion de l'espace domestique. Toutefois, les signes de cette extraversion, en tant qu'expression de l'urbanité différentielle des groupes sociaux, restent plus ou moins contrôlés (F. Navez-Bouchanine, 1994). Les modalités de gestion de l'extraversion de l'habitat renvoient à une recherche d'équilibre entre le besoin de protéger l'espace domestique et de lui conférer un caractère urbain. L'extraversion de l'habitat, processus général qui porte sur les espaces intermédiaires, notamment la façade et les accès, signifie en fait un rapport nouveau de l'habitant à l'espace urbain. L'habitant s'intéresse ainsi davantage au devenir de l'espace urbain et s'investit de plus en plus dans sa mise en valeur. L'extraversion de l'habitat implique, dans la formalisation des espaces intermédiaires entre le chez-soi et l'urbain, très peu de séquences de protection, contrairement à l'espace domestique traditionnel où elles étaient multiples (seuil, rideau, chicane, skiffa, cour d'entrée, impasse, etc.).

L'habitat traditionnel totalement introverti présentait une façade intérieure qui encadrait le patio et contrastait avec la sobriété extérieure du bâti. Ce schème a d'ailleurs considérablement structuré les modalités d'appropriation de la population rurale venue en ville au moment de l'indépendance. Les pratiques de fermeture de l'habitat s'étaient alors généralisées : surélévation des garde-corps de balcon et des clôtures, protection des ouvertures par des rideaux, des roseaux ou de la bâche, etc. Aujourd'hui, un processus inverse « retourne » progressivement l'espace domestique vers l'extérieur. Les habitants procèdent en effet de plus en plus à la réouverture de la façade sur l'espace urbain en la chargeant des signes de l'urbanité, de l'ostentation et de la distinction. En atteste la diversité des matériaux utilisés et des formes de décoration mises en œuvre.

Dans ces processus de réouverture de la façade, le balcon, qui constitue un autre lieu typique partiel de référence urbaine, joue un rôle fondamental dans l'expression de l'urbanité des habitants. Bien que les pratiques sur ce lieu se limitent le plus souvent à l'aération de la literie et occasionnellement à regarder le cortège d'un mariage, le balcon, conçu comme élément de décor s'est généralisé dans tous les édifices au point de courir sur l'ensemble du volume des constructions. Même dans les cités de recasement, où les façades sont très étroites parce que l'habitat y était initialement introverti, les résidents intègrent de petits balcons en fer forgé qui, dans ce cas, ont également pour objectif de personnaliser la maison et d'exprimer clairement les moyens consentis pour améliorer l'espace domestique. Paradoxalement, l'ouverture de l'espace domestique sur l'urbain s'accompagne de l'édification de clôtures autour de l'espace privé qui ont l'allure de véritables murailles. Cette pratique, qui dans certains cas peut être une forme d'expression de la volonté de maîtriser cette extraversion, renvoie surtout à un souci sécuritaire spécifique au milieu urbain et à la situation de ces dernières années. Outre l'édification de clôtures, l'installation de barreaux sur les ouvertures, le blindage des portes, la mise en place de système d'alarme, etc., sont devenues des pratiques sécuritaires généralisées dont l'importance reste fonction de la richesse du groupe social concerné.

\subsection{Des normes urbaines pour promouvoir les nouvelles polarités.}

La morphologie des quartiers illicites est très différente selon qu'ils soient anciens ou récents. Dans le premier cas, le bâti est très dense tandis que dans le deuxième, il a une forme plus aérée grâce à un réseau de voiries mieux calibré. Ainsi, l'occupation et l'appropriation des nouveaux quartiers, que ce soit ceux produits illicitement ou ceux des lotissements 
réglementés, sont fonction de l'accessibilité aux voitures. Même les transformations que connaissent les cités de recasement, en particulier celles qui consistent à organiser l'extension de la maison sur le domaine public, tiennent compte de la circulation automobile. Il y a ainsi une sorte de rectification progressive de l'espace public dans les quartiers auto-construits prenant en compte l'accessibilité des voitures, laquelle est considérée comme un attribut de l'espace urbain. Ainsi les dessertes ont des gabarits de plus en plus importants et la présence d'aires de stationnement et de garages se généralise dans l'habitat grâce à la réorganisation des accès.

L'introduction d'une activité économique dans l'espace domestique n'est pas étrangère à l'intégration de l'élément voiture dans la façon de penser et de produire l'espace urbain. Cependant, la volonté de valoriser les quartiers, de les faire entrer dans un cadre légal et d'améliorer une image qui renvoie aux oppositions quartiers riches organisés et quartiers pauvres anarchiques, sont les véritables moteurs de cette façon de produire la ville. Cette dynamique a fini par produire un véritable tissu économique dans les nouvelles périphéries informelles, économie qui peu à peu s'organise et s'adapte aux besoins de sa clientèle. On voit ainsi apparaître de nouvelles polarités urbaines dont l'offre de biens et de services s'avère large et répartie sur l'ensemble de l'agglomération. Ces polarités nouvelles sont à la fois une réponse à une demande sociale réelle à l'échelle de l'agglomération générant une forte attractivité vers ces quartiers et un biais par lequel ces quartiers organisent leur valorisation. Animés par des stratégies qui visent un meilleur fonctionnement de cette économie familiale, les efforts des habitants et leurs actions collectives auprès des pouvoirs publics ont permis d'introduire progressivement les commodités nécessaires au fonctionnement du quartier et de ces nouvelles centralités (alimentation en électricité, gaz, éclairage public, etc.) et des normes pour asseoir, voire promouvoir ces nouvelles polarités.

\subsection{Mobilités résidentielles et mobilités sociales}

Afin de traduire l'ensemble des stratégies urbaines, l'analyse gagnerait à intégrer celles des mobilités résidentielles. A l'origine de son processus d'urbanisation, la population de l'exode rural sur Alger n'a pas d'autres possibilités de logement que la maison commune. Cette dernière est considérée d'emblée par les habitants comme une étape transitoire dans leur itinéraire résidentiel qu'ils cherchent désespérément à faire coïncider avec leur ascension sociale car cet habitat est vécu comme dégradant. Si les habitants de la Casbah et ceux des autres maisons communes du parc immobilier vétuste peuvent espérer obtenir un logement en HLM et de ce fait valoriser dans une certaine mesure leur image sociale, les résidents des HLM et des cités de recasement, en revanche, ont moins de chance. Leur itinéraire résidentiel et leur mobilité sociale sont contrariés. Les compétences des habitants des HLM et des cités de recasement ont épuisé toutes les possibilités que peut offrir leur habitat pour protéger les intimités et vivre décemment. Dans les cités de recasement, les processus de densification et d'appropriation de l'espace public ont atteint un seuil de dégradation et de dévalorisation très élevé. De même, dans les HLM, toutes les combinaisons de division/dédoublement, de translation sur les espaces extérieurs et de polyvalence des pièces, effectuées par les habitants pour tenter de gérer la pénurie d'espace ont atteint leurs limites. Ces groupes sociaux se retrouvent souvent piégés dans une situation sociale précaire dont les issues restent minces. La population de ces quartiers tente alors de préserver, tant bien que mal, certaines sociabilités, notamment à travers les associations. Paradoxalement, plus la mobilité résidentielle est contrariée, plus les couples dont les revenus le permettent se désolidarisent financièrement de la famille élargie et tentent d'échapper à l'étau du quartier. Les pratiques de spécialisation des pièces, de dédoublement et de desserrement de l'espace habitable du HLM ne concernent que les groupes sociaux qui ont eu la chance de procéder à leur ascension 
sociale par le truchement de la formation des enfants et des débouchés professionnels qu'elle leur offre. Le reste des familles s'enlise dans une situation sociale où le nombre d'actifs en leur sein diminue considérablement face à une progression effarante des personnes à charge (chômeurs, femmes au foyer, personnes âgées ou en bas âge).

Inversement, les pratiques d'appropriation des habitants du reste du parc immobilier étudié32 font apparaître une trajectoire sociale dont la logique est vraisemblablement ascensionnelle. Effectivement, les nouveaux modes de sociabilité, les pratiques de distinction sociale et les stratégies familiales, notamment celles qui consistent à accumuler un capital familial, mettent en évidence les efforts des couches moyennes pour se hisser dans l'échelle sociale et pour traduire à travers leur habitat une position sociale acquise ou prétendue. Les modalités d'appropriation se manifestent alors par la taille de la maison construite, par l'importance des espaces de la représentation et de l'ostentation et par un sur investissement en matière d'ameublement, d'équipement et de décor et se placent dans le champ de la distinction sociale. Les stratégies des groupes sociaux pour rester dans cette logique ascendante et l'affirmer se déploient d'abord au sein de la famille, même si celle-ci intègre un processus inverse qui est celui de l'autonomie des couples. Une des stratégies familiales la plus remarquable est celle qui consiste à organiser dans l'habitat un espace d'accumulation du capital familial. Cette pratique, en tant que biais par lequel s'effectue l'ascension sociale, est devenue un modèle dominant. Même dans les cités de recasement, les habitants ouvrent des échoppes, là où la relation à l'espace public le permet.

\section{Conclusion}

A travers l'émergence de nouveaux types d'habitat, se manifeste également la recherche de modèles qui témoignent d'une forme d'urbanité ou d'une intégration urbaine plus importante. Les nouvelles pratiques qui apparaissent, également à l'échelle urbaine, traduisent une pensée collective basée sur une réinterprétation active des règles de l'urbanistique. Elles donnent lieu à de nouvelles normes urbaines dont l'objectif et d'écarter tout risque de marginalisation et d'œuvrer à une véritable intégration. Ces normes trouvent leur traduction à la fois dans les traits réguliers et facilement repérables de l'espace urbain produit, notamment l'emprise des voies de desserte et la qualité des espaces publics, et à la fois, dans les modes d'articulation de l'espace domestique avec le domaine urbain, dont l'expression la plus manifeste reste l'extroversion de l'habitat. La mise en pratique des représentations de l'espace, traduite par les phénomènes d'appropriation, en même temps qu'elle exprime des mutations au sein des modèles socioculturels, ouvre le champ à la compréhension des transformations en cours dans la société algérienne. Parmi ces mutations, apparaissent dans de larges milieux la nucléarisation des ménages et l'émergence du couple comme entité économiquement et socialement autonome. Cette nouvelle position du couple octroie à la femme un statut nouveau au sein de la famille qui s'écarte sensiblement du modèle traditionnel et déplace son activité du lieu domestique à l'espace urbain.

La dynamique socioculturelle actuelle en même temps qu'elle induit la disparition du caractère aliénant de la communauté sur l'individu et son émergence met en place de nouvelles sociabilités qui sont à la base des formes d'organisation du type associatif. Si la raison d'être de ces nouvelles formes d'organisation se trouve dans la revalorisation de l'image du quartier et son intégration à la ville, elle est aussi et surtout, dans une conscience naissante

\footnotetext{
32 Habitat individuel dense, lotissements modestes de l'époque coloniale, nouveaux lotissements, habitat individuel à standing moyen, immeubles haussmanniens, résidences du mouvement moderne, ensembles récents de standing.
} 
de la citoyenneté et de son rôle dans l'espace public. Il n'en reste pas moins que ces émergences multiples subissent le contrecoup d'un contrôle social sévère, qui forme une sorte de chape de plomb sur l'ensemble du corps social et le pousse à fonctionner dans une ambivalence entre ce qui est montré et ce qui reste caché.

\section{Bibliographie.}

BONETTI M., 1994, Habiter : le bricolage imaginaire de l'espace, Marseille, Hommes \& Perspectives, coll. Re-connaissances, $229 \mathrm{p}$.

BOURDIEU P., 1972, Esquisse d'une théorie de la pratique, Genève, Droz, coll. Travaux de droit, d'économie, de sociologie et de sciences politiques.

BOURDIEU P., 1993, «Les Effets de lieu », in BOURDIEU P., (dir.), La Misère du monde, Paris, Seuil, pp. 249-261.

CROIZE J.-C., FREY J.-P., PINON P., 1991, Recherches sur la typologie et les types architecturaux, actes de la table ronde internationale au centre de recherche sur l'habitat, Paris, L'Harmattan.

DEVILLERS C., 1974, «Typologie de l'habitat et morphologie urbaine », in Architecture d'Aujourd'hui, n¹74, juillet-août 1974.

FREY J.-P., 1990, «Conclusion », in Regards sur le lotissement, séminaire de la délégation à la recherche et à l'innovation, Paris, éditions du service technique de l'urbanisme.

FREY J.-P., 1986, La Ville industrielle et ses urbanités. La distinction ouvriers/employés. Le Creusot 1870-1930, Liège, Pierre Margada, coll. Architecture et recherche, 386 p.

HAUMONT N., 2001, (1 ${ }^{\text {̀re }}$ éd. 1966), Les Pavillonnaires, Paris, L'Harmattan, coll. Habitat et sociétés, $150 \mathrm{p}$.

BERRY-CHIKHAOUI I., DEBOULET A. (dir.), 2000, Les Compétences des citadins dans le Monde arabe. Penser, faire et transformer la ville, Paris, Karthala, coll. Hommes et sociétés, $406 \mathrm{p}$.

DE MAISONSEUL J., 1980, «Djenan El Hassan. Relations espace/temps : ou redécouverte de l'échelle humaine chez Roland Simounet », in Technique \& Architecture, $\mathrm{n}^{\circ} 329$, févriermars 1980, p. 65

LEFEBVRE H., 1970, La Révolution urbaine, Paris, Gallimard, coll. Idées, 248 p.

NAVEZ-BOUCHANINE F., 1994, «Que faire des modèles d'habiter ? », in Architecture \& Comportement, volume 10, $\mathrm{n}^{\circ} 3$, pp. 231-344.

PRENANT A., 2002, «L'Aggravation des contrastes sociaux à travers une extension spatiale et un freinage démographique différencié dans la nébuleuse urbaine d'Alger», Alger, lumières sur la ville, actes du colloque du 4 au 6 mai à l'EPAU, vol. II, pp. 386-404.

SEMMOUD N., 2001, Les Stratégies d'appropriation de l'espace à Alger, préface de FREY (Jean-Pierre), Paris, L'Harmattan, coll. Histoire et perspectives méditerranéennes, 270 p. 